Kompass

Autoimmun

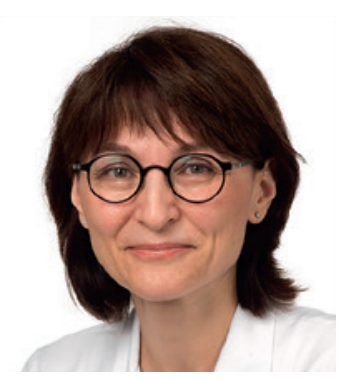

\title{
Polygener Risikoscore hilft bei der Diagnose der Spondylitis akylosans
}

\author{
Carina Mihai
}

Universitätsklinikum Zürich, Klinik für Rheumatologie, Zürich, Schweiz

Abstract aus Li Z, Wu X, Leo PJ, et al.: Polygenic risk scores have high diagnostic capacity in ankylosing spondylitis. Ann Rheum Dis 2021, in press. DOl: 10.1136/annrheumdis-2020-219446

\section{Keywords}

ankylosing · genetic · low back pain · magnetic resonance imaging $\cdot$ polymorphism $\cdot$ spondylitis

\begin{abstract}
Objective: We sought to test the hypothesis that Polygenic Risk Scores (PRSs) have strong capacity to discriminate cases of ankylosing spondylitis (AS) from healthy controls and individuals in the community with chronic back pain.
\end{abstract}

Methods: PRSs were developed and validated in individuals of European and East Asian ethnicity, using data from genome-wide association studies in 15585 AS cases and 20452 controls. The discriminatory values of PRSs in these populations were compared with other widely used diagnostic tests, including C-reactive protein (CRP), HLA-B27 and sacroiliac MRI.

Results: In people of European descent, PRS had high discriminatory capacity with area under the curve (AUC) in receiver operator characteristic analysis of 0.924 . This was significantly better than for HLA-B27 testing alone (AUC $=0.869)$, MRI (AUC $=0.885)$ or $C$ reactive protein $(A U C=0.700)$. PRS developed and validated in individuals of East Asian descent performed similarly (AUC = 0.948). Assuming a prior probability of AS of $10 \%$ such as in patients with chronic back pain under 45 years of age, compared with HLA-B27 testing alone, PRS provides higher positive values for $35 \%$ of patients and negative predictive values for $67.5 \%$ of patients. For PRS, in people of European descent, the maximum positive predictive value was $78.2 \%$ and negative predictive value was $100 \%$, whereas for HLA-B27, these values were $51.9 \%$ and $97.9 \%$, respectively.

Conclusions: PRS have higher discriminatory capacity for AS than CRP, sacroiliac MRI or HLA-B27 status alone. For optimal performance, PRS should be developed for use in the specific ethnic groups to which they are to be applied.

(c) Author(s) (or their employer(s)) 2021 


\section{Transfer in die Praxis}

\section{Hintergrund}

Genetische Faktoren sind die wichtigsten Determinanten für das Risiko, an Spondylitis ankylosans (SA) zu erkranken. Der HLA-B27Test wird bei der Diagnose der SA häufig gebraucht, erfasst jedoch nur einen moderaten Anteil ( $20 \%)$ des gesamten genetischen Risikos für die Krankheit, die auf $\geq 90 \%$ insgesamt geschätzt wird, was auf eine wesentliche Nicht-Major Histocompatibility Complex (MHC)-Komponente hinweist [1]. Diese Komponente wird durch Tausende gängiger genetischer Varianten bestimmt, von denen jede nur eine sehr geringe Wirkung hat. Polygenic Risk Scores (PRS) verwenden Kombinationen von Hunderten bis Tausenden genetischer Varianten, um das genetische Krankheitsrisiko einer Person zu quantifizieren [2]. Im Gegensatz zu HLA-B27-Tests, die entweder zu einem positiven oder einem negativen Befund führen, handelt es sich bei den PRS um kontinuierliche Messungen. PRS-Tests können unter Verwendung von Daten von jedem dichten SNP-Mikroarray (SNPs = Einzelnukleotidpolymorphismen) durchgeführt werden und haben einen besonders starken prädiktiven Wert für niederfrequente Erkrankungen mit hoher Heritabilität wie SA [3].

\section{Ergebnisse der Studie}

Ein internationales Team entwickelte und validierte einen PRS für SA in einer großen multi-ethnischen Fall-Kontroll-Studie und verglich seine Leistung mit Standard-Screening- oder Standard-diagnostischen Tests. Es wurden insgesamt über 16000 Patienten mit SA eingeschlossen, die die modifizierten New York-Kriterien für SA erfüllten [4]. Die Gruppe der gesunden Kontrollpersonen umfasste über 20000 Personen.

Es wurde eine genomweite Assoziationsstudie (GWAS) durchgeführt: DNA-Proben wurden mit dem Illumina Core-Exome SNP Ge- notyping Microarray gemäß den Empfehlungen des Herstellers genotypisiert. Es erfolgte eine komplexe Selektion der Patienten und Kontrollpersonen durch Standard-Qualitätskontrollmaßnahmen (Identifizierung und Ausschluss von kryptischen Proben, von Proben mit einer abweichenden Heterozygotie-Rate oder Fehlbildung $>5 \%$ ) und Entfernung von Proben mit Problemen (SNPs mit einer Genotyping Missing-Rate > 2\%, einem $p$-Wert des Hardy-Weinberg-Gleichgewichtstests $<1 \times 10^{-6}$ oder mit einer Allelfrequenz < 1\%). Schlussendlich schloss die Kohorte 8244 Fälle und 14274 Kontrollen westeuropäischer Abstammung, 6001 Fälle und 4493 Kontrollen ostasiatischer (chinesischer) Abstammung und 1340 Fälle bzw. 1685 Kontrollen türkischer und iranischer Herkunft ein. Daten über HLA-B27 und Geschlecht wurden unter Verwendung spezifischer genetischer Instrumente aus den GWAS-Resultaten erhoben. Alle Datensätze wurden schließlich in eine europäische Herkunftskohorte und eine ostasiatische Kohorte zusammengeführt.

Es erfolgte eine komplexe und rigorose statistische Analyse der Daten. Die Testdiskriminierungskapazität des PRS wurde mittels Analyse der Receiver Operating Characteristic (ROC)-Kurve nach 10-facher Kreuzvalidierung in dieser Fall-Kontroll-Kohorte gerechnet, verglichen wurden die Flächen unter der Kurve (AUC). Positive (PPV) und negative prädiktive Werte (NPV) wurden dann für PRS-Zentile berechnet, wobei verschiedene frühere Wahrscheinlichkeiten von SA angenommen wurden. Die kontinuierliche Netto-Reklassifizierungsverbesserung (NRI), eine Statistik, die darauf abzielt, Unterschiede in der Klassifikationsleistung verschiedener Modelle zu quantifizieren, wurde verwendet, um die Genauigkeit der diagnostischen Zuordnung durch HLA-B27-Tests und PRS zu vergleichen. Die Resultate zeigten eine Überlegenheit des PRS gegenüber HLAB27. Der PRS zeigte die höchste Testdiskriminierungskapazität, mit
Abb. 1. ROC-Kurve der Leistung von PRS (violette Linie, $A \cup C=0,924)$, HLA-B27 (türkisfarbene Linie, $A \cup C=0,869$ ), PRS ohne MHC (grüne Linie, $A \cup C=0,782)$ und nur genomweiten signifikanten Loci (rote Linie, $A \cup C=0,659$ ) (aus: Li et al., Ann Rheum Dis 2021, DOl: 10.1136/annrheumdis-2020-219446).

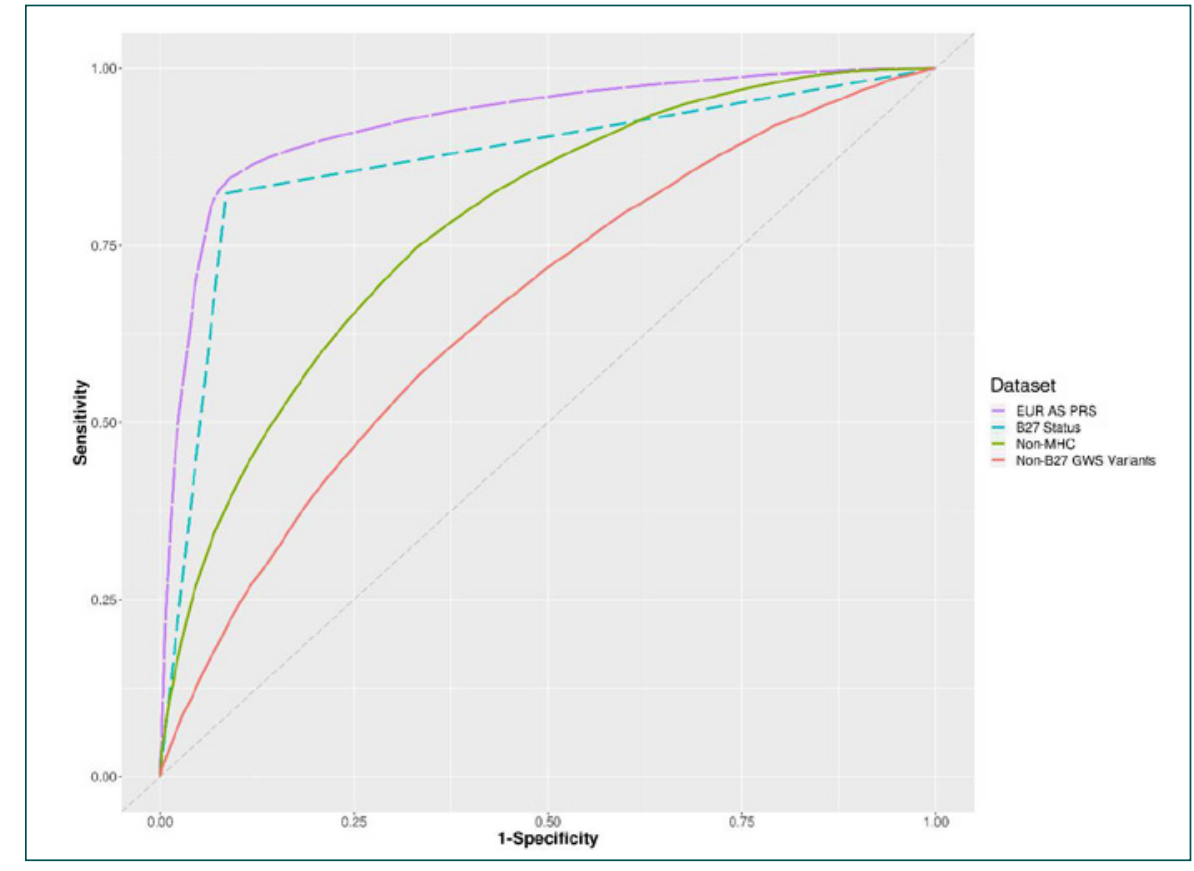


einer AUC von 0,924 (95\%-Konfidenzintervall (KI) 0,920-0,928) (Abb. 1). Die AUC des HLA-B27-Tests allein betrug 0,869 (95\%-KI 0,865-0,874), was statistisch signifikant weniger diskriminierend war als der PRS $\left(p<2,2 \times 10^{-16}\right)$. Ein PRS, der nur Nicht-MHC-SNPs enthielt, schnitt weniger gut ab (AUC 0,782). Darüber hinaus war die NRI positiv (0,717, 95\%-KI 0,692-0,743), was bestätigt, dass der PRS eine Verbesserung gegenüber HLA-B27 allein darstellt.

Der PRS schnitt in der Allgemeinbevölkerung deutlich besser ab als HLA-B27-Tests allein, wobei der PPV der 8\% der Allgemeinbevölkerung, die HLA-B27 tragen, 5\% beträgt, verglichen mit dem Spitzen-PPV des PRS von $15,1 \%$.

Dasselbe konnte im Vergleich zur Diagnose der SA durch lediglich Kernspinresonanz der Iliosakralgelenke (ISG-MRI) gesagt werden, die in einer Studie eine Sensitivität von 85\% und eine Spezifität von 92\% hatte, was mit einer AUC von 0,885 korreliert [5]. C-reaktives Protein (CRP) hat eine berichtete Sensitivität von $50 \%$ und eine Spezifität von 80\% für die Krankheit (AUC = 0,7) [6].

\section{Fazit für die Praxis}

Diese elegante Studie entwickelte und validierte einen PRS, der sowohl in AUC-als auch in kontinuierlichen NRI-Analysen besser als der HLA-B27-Test abschnitt, unabhängig von der Prävalenz von SA unter den getesteten Personen. Der PRS hatte auch eine größere Unterscheidungskraft für SA als MRI-Scans oder CRP-Tests, entweder allein oder in Kombination.

Der PRS könnte verwendet werden, um Patienten mit chronischen Rückenschmerzen für SA zu screenen, um Patienten mit erhöhtem SA-Risiko für die Überweisung an die Sekundärversorgung zu iden- tifizieren und um die Diagnose der SA zu unterstützen. Der aktuelle PRS kann einen prognostischen Wert bei der Unterscheidung der 16-24\% Fälle von nichtradiologischer Spondylarthritis haben, die wahrscheinlich später eine SA entwickeln werden. Die Kosteneffektivität des hier vorgestellten PRS-Tests muss in weiteren Studien bestätigt werden.

\section{Disclosure Statement}

Hiermit erkläre ich, dass keine Interessenkonflikte in Bezug auf den vorliegenden Kommentar bestehen.

\section{Literatur}

1 Brown MA, Crane AM, Wordsworth BP: Genetic aspects of susceptibility, severity, and clinical expression in ankylosing spondylitis. Curr Opin Rheumatol 2002;14:354-360.

2 Ellinghaus D, Jostins L, Spain SL, et al.: Analysis of five chronic inflammatory diseases identifies 27 new associations and highlights disease-specific patterns at shared loci. Nat Genet 2016:48:510-518.

3 Wray NR, Yang J, Goddard ME, et al.: The genetic interpretation of area under the ROC curve in genomic profiling. PLOS Genet 2010;6:e1000864.

4 van der Linden S, Valkenburg HA, Cats A: Evaluation of diagnostic criteria for ankylosing spondylitis. A proposal for modification of the New York criteria. Arthritis Rheum 1984;27:361-368

5 Diekhoff T, Hermann K-GA, Greese J, et al.: Comparison of MRI with radiography for detecting structural lesions of the sacroiliac joint using CT as standard of reference: results from the SIMACT study. Ann Rheum Dis 2017;76:1502-1508.

6 Rudwaleit M, van der Heijde D, Khan MA, et al.: How to diagnose axial spondyloarthritis early. Ann Rheum Dis 2004;63:535-543.

Korrespondenz an:

Carmen-Marina Mihai, carmen-marina.mihai@usz.ch 